\title{
Polariton dynamics in strongly interacting quantum many-body systems
}

\author{
A. Camacho-Guardian $\odot,{ }^{1}$ K. Knakkergaard Nielsen $\odot,{ }^{1}$ T. Pohl,${ }^{1}$ and G. M. Bruun ${ }^{1,2}$ \\ ${ }^{1}$ Department of Physics and Astronomy, Aarhus University, Ny Munkegade, 8000 Aarhus C, Denmark \\ ${ }^{2}$ Shenzhen Institute for Quantum Science and Engineering and Department of Physics, \\ Southern University of Science and Technology, Shenzhen 518055, China
}

(Received 3 September 2019; accepted 3 April 2020; published 29 April 2020)

\begin{abstract}
We develop a theory for light propagating in an atomic Bose-Einstein condensate in the presence of strong interactions. The resulting many-body correlations are shown to have profound effects on the optical properties of this interacting medium. For weak atom-light coupling, there is a well-defined quasiparticle, the polaronpolariton, supporting light propagation with spectral features differing significantly from the noninteracting case. The damping of the polaron-polariton depends nonmonotonically on the light-matter coupling strength, initially increasing and then decreasing. This gives rise to an interesting crossover between two quasiparticles: a bare polariton and a polaron-polariton, separated by a complex and lossy mixture of light and matter.
\end{abstract}

DOI: 10.1103/PhysRevResearch.2.023102

\section{INTRODUCTION}

The ability to prepare, control, and probe cold matter systems via external light fields is at the heart of modern developments in atomic physics, quantum optics, many-body physics, and quantum technologies. Here, electromagnetically induced transparency (EIT) presents a particularly powerful approach to achieve strong light-matter coupling at greatly reduced losses. This effect opens up numerous applications, from cooling [1] and trapping [2] techniques, to the realization of quantum memories [3] and ultraslow propagation of light in the form of dark-state polaritons [4]. EIT has been observed in a wide variety of media including hot atomic vapors [5], cold atomic gases [6-8], Rydberg gases [9], and solids [10]. While many of these applications utilize relatively simple optical media, coupling photons to strongly interacting quantum many-body systems would open the door to quantum nonlinear optics, based on their rich spectrum of strong-correlation phenomena [11,12]. Indeed, understanding light propagation in strongly correlated environments remains a problem of great scientific and technological significance that is currently attracting increasing interest in both atomic [13-16] and solid-state [17,18] settings. Although the promise of combining EIT and strong particle interactions is widely recognized [19], the effects of environmental coupling on the dynamics of single slow-light quanta remain to be understood. Here we address this problem by developing a nonperturbative theory for the quantum dynamics of a dark-state polariton in Bose-Einstein condensates (BECs), and we explore the effects of strong interactions between its spin-wave component and the surrounding condensate. Interestingly, these

Published by the American Physical Society under the terms of the Creative Commons Attribution 4.0 International license. Further distribution of this work must maintain attribution to the author(s) and the published article's title, journal citation, and DOI. interactions lead to the formation of polaron quasiparticles [20,21], i.e., phonon dressed impurities, while the formation of dark-state polaritons corresponds to photon dressing of the same impurity state. The developed theory makes it possible to explore the competition between the creation of these two quasiparticles. While this generally causes the formation of complex light-matter states with substantial environmental dissipation, we identify regimes in which light propagation can be understood in terms of a well-defined quasiparticle that features reduced decoherence and inherits the properties of both quasiparticle excitations. This polaron-polariton state is shown to have a narrowed EIT linewidth and an even lower group velocity compared to the bare slow-light polariton. Our study establishes a general theoretical framework for quantum optics in strongly interacting systems and will provide a guide for achieving coherent interfacing and photon-photon interactions in atomic gases and semiconductor materials.

\section{MODEL}

We consider atoms of mass $m$ with three internal states $|b\rangle,|e\rangle$, and $|c\rangle$. A quantized probe beam couples the $|b\rangle$ and $|e\rangle$ states with a single-photon coupling $g$, whereas a classical control field couples the $|e\rangle$ and $|c\rangle$ states with Rabi frequency $\Omega$, forming a so-called $\Lambda$-scheme. Within the rotating wave approximation, the Hamiltonian can be written as

$$
\begin{aligned}
& H=\sum_{\mathbf{p}}\left[\epsilon_{\mathbf{p}} b_{\mathbf{p}}^{\dagger} b_{\mathbf{p}}+\epsilon_{\mathbf{p}}^{(e)} e_{\mathbf{p}}^{\dagger} e_{\mathbf{p}}+\epsilon_{\mathbf{p}}^{(c)} c_{\mathbf{p}}^{\dagger} c_{\mathbf{p}}+c p \gamma_{\mathbf{p}}^{\dagger} \gamma_{\mathbf{p}}\right] \\
&+\sum_{\mathbf{p}}[ {\left[\Omega e_{\mathbf{p}}^{\dagger} c_{\mathbf{p}-\mathbf{k}_{\mathrm{cl}}}+\sum_{\mathbf{q}} g e_{\mathbf{p}+\mathbf{q}}^{\dagger} b_{\mathbf{p}} \gamma_{\mathbf{q}}+\text { H.c. }\right] } \\
&+\sum_{\mathbf{p}, \mathbf{p}^{\prime}, \mathbf{q}}\left[V_{\mathrm{B}}(q) b_{\mathbf{p}+\mathbf{q}}^{\dagger} b_{\mathbf{p}^{\prime}-\mathbf{q}}^{\dagger} b_{\mathbf{p}^{\prime}} b_{\mathbf{p}} / 2\right. \\
&\left.+V(q) b_{\mathbf{p}+\mathbf{q}}^{\dagger} c_{\mathbf{p}^{\prime}-\mathbf{q}}^{\dagger} c_{\mathbf{p}^{\prime}} b_{\mathbf{p}}\right],
\end{aligned}
$$




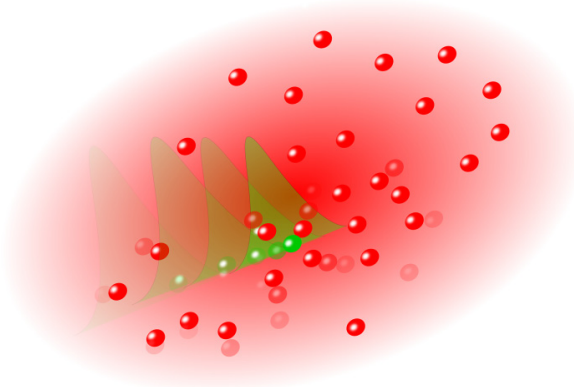

FIG. 1. Light propagating as a polaron-polariton in a BEC.

where the operators $b_{\mathbf{p}}^{\dagger}, c_{\mathbf{p}}^{\dagger}$, and $e_{\mathbf{p}}^{\dagger}$ create an atom with momentum $\mathbf{p}$ and kinetic energy $\epsilon_{\mathbf{p}}=p^{2} / 2 m$ in the atomic state $|b\rangle,|c\rangle$, and $|e\rangle$, respectively. The atomic states are such that $\epsilon_{\mathbf{p}}^{(e)}=\epsilon_{\mathbf{p}}+\epsilon_{e}$ and $\epsilon_{\mathbf{p}}^{(c)}=\epsilon_{\mathbf{p}}+\epsilon_{c}+\omega_{\mathrm{cl}}$ with $\epsilon_{e / c}$ their bare state energies, respectively. Here $\epsilon_{\mathbf{p}}^{(e)}$ includes the Lamb shift due to the coupling $g$ to the $|b\rangle \otimes|\gamma\rangle$ continuum. The operator $\gamma_{\mathbf{p}}^{\dagger}$ creates a photon with momentum $\mathbf{p}$ and kinetic energy $c p$ with $c$ the speed of light in a vacuum. The second line of Eq. (1) describes the coupling between the atoms and the probe photons as well as the classical control field. Note that the classical field with wave vector $\mathbf{k}_{\mathrm{cl}}\left(\omega_{\mathrm{cl}}=c\left|\mathbf{k}_{\mathrm{cl}}\right|\right)$ decreases the momentum of the $|c\rangle$ atoms by $\mathbf{k}_{\mathrm{cl}}$ compared to the $|b\rangle$ and $|e\rangle$ atoms. The interaction $V_{\mathrm{B}}(q)=4 \pi a_{B} / m$ describes the interaction between two atoms in state $|b\rangle$, and $V(q)=$ $\mathcal{T}_{v}=4 \pi a / m$ denotes the interaction between a $|b\rangle$ - and a $|c\rangle$ state atom. Both interactions are short range and accurately characterized by the scattering lengths $a_{\mathrm{B}}$ and $a$ respectively. We use units where the system volume and $\hbar$ are both one. The $|b\rangle$ atoms form a weakly interacting three-dimensional BEC with density $n=k_{n}^{3} / 6 \pi^{2}$ and $0<k_{n} a_{\mathrm{B}} \ll 1$. The excitation spectrum of the BEC is given by Bogoliubov theory, i.e., $E_{\mathbf{p}}=$ $\sqrt{\epsilon_{\mathbf{p}}\left(\epsilon_{\mathbf{p}}+2 \mu_{B}\right)}$ with $\mu_{B}=4 \pi a_{\mathrm{B}} n / m$ its chemical potential.

As illustrated in Fig. 1, we consider a photon with momentum $\mathbf{k}$ propagating inside the BEC. This excites an atom out of the BEC and into the $|e\rangle$ and $|c\rangle$ states via the $\Lambda$ scheme. We focus on the case of a small density of $|c\rangle$ atoms so that they can be regarded as impurities in the BEC. Strictly speaking, this corresponds to the limit of a single photon propagating through the BEC, but in analogy with the case of impurities in atomic gases in the absence of light [20-22], we expect this picture to be accurate as long as the density of the $|c\rangle$ atoms is much smaller than that of the BEC, which consequently acts as a particle reservoir. This furthermore means that we can ignore $|e\rangle-|e\rangle,|e\rangle-|c\rangle$, and $|c\rangle-|c\rangle$ interactions since the densities of these states are so low. Finally, the scattering length $a$ describing the $|b\rangle-|c\rangle$ interaction is taken to be tuneable so that the unitary regime $k_{n}|a| \gtrsim 1$ of strong interaction can be reached.

\section{DISCUSSION}

Before we plunge into detailed calculations, let us discuss the main physical concepts and results. The system combines two paradigmatic quasiparticles, the dark-state polariton giving rise to EIT in the absence of atomic interactions, and the Bose polaron emerging due to (a)
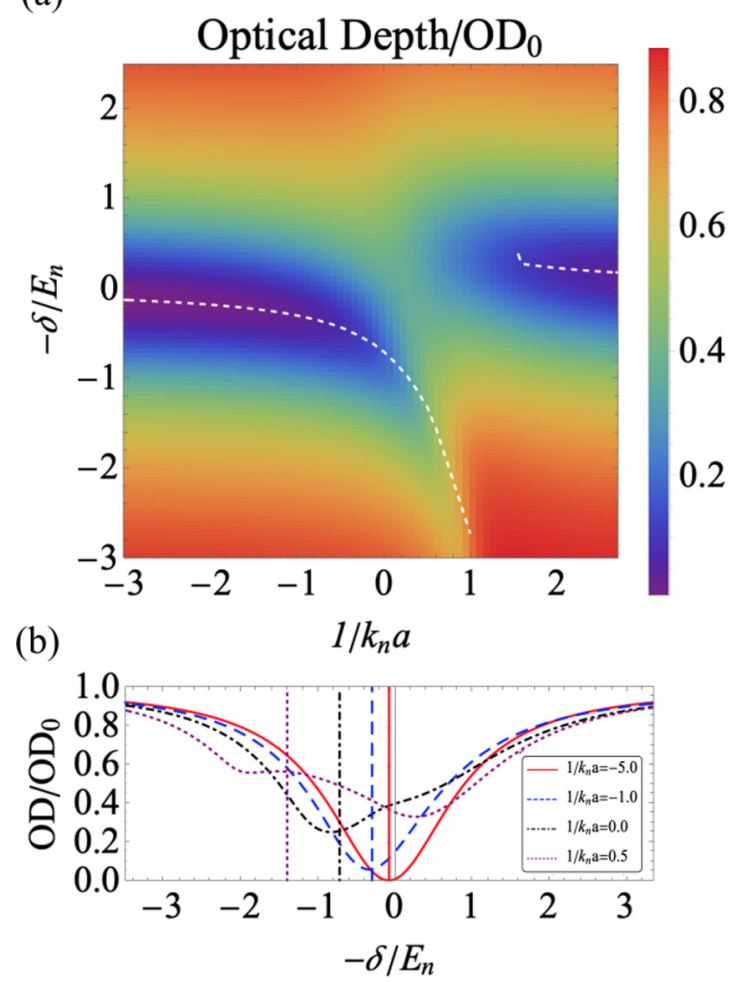

FIG. 2. (a) Optical depth as a function of the two-photon detuning and the atomic interactions. (b) Cross sections of the optical depth for several values of the inverse of the interaction strength. The vertical lines give the polaron energy in the absence of light.

interactions when there is no light. The polariton wave function is $\left|D_{\mathbf{k}}\right\rangle=-\cos \theta \gamma_{\mathbf{k}}^{\dagger}|\mathrm{BEC}\rangle+\sin \theta c_{\mathbf{k}-\mathbf{k}_{\mathrm{c}}}^{\dagger}|\mathrm{BEC}\rangle$ with $|\mathrm{BEC}\rangle$ the wave function of the BEC of $|b\rangle$ atoms and $\cos ^{2} \theta=1 /\left(1+g^{2} n /|\Omega|^{2}\right) \quad[4,23]$. The polaron wave function can, on the other hand, be written as $\left|\psi_{P, \mathbf{k}-\mathbf{k}_{\mathrm{cl}}}\right\rangle=\left(\sqrt{Z_{P}} c_{\mathbf{k}-\mathbf{k}_{\mathrm{cl}}}^{\dagger}+\sum_{\mathbf{q}} \psi_{q} c_{-\mathbf{q}+\mathbf{k}-\mathbf{k}_{\mathrm{cl}}}^{\dagger} \beta_{\mathbf{q}}^{\dagger}\right)|\mathrm{BEC}\rangle$, which describes the impurity dressed by Bogoliubov excitations created by $\beta_{\mathbf{q}}^{\dagger}$ [24-26]. We have introduced the quasiparticle residue $Z_{P}$ of the polaron and $\psi_{q}$ are expansion coefficients. Now, it is tempting to assume that the presence of both strong light coupling and atomic interactions would lead to the formation of a polaron-polariton of the form

$$
\left|D_{\mathbf{k}}^{P}\right\rangle=-\cos \theta \gamma_{\mathbf{k}}^{\dagger}|\mathrm{BEC}\rangle+\sin \theta\left|\psi_{P, \mathbf{k}-\mathbf{k}_{\mathrm{cl}}}\right\rangle,
$$

which is a quasiparticle encompassing simultaneously the polariton and polaron features by replacing the noninteracting impurity $c_{\mathbf{k}-\mathbf{k}_{\mathrm{c}}}^{\dagger}|\mathrm{BEC}\rangle$ state by the polaron $\left|\psi_{P, \mathbf{k}-\mathbf{k}_{\mathrm{cl}}}\right\rangle$. We shall show that although this is an accurate description in certain regimes, it breaks down in other regimes in favor of a complex light-matter quantum state.

Figure 2(a) summarizes our results. It shows the optical depth of a BEC as a function of the two-photon detuning $\delta=$ $\epsilon_{c}+\omega_{\mathrm{cl}}-c k$ and the interaction strength $1 / k_{n} a$. First, we see a pronounced minimum in the optical density, which for weak interactions is almost zero. The minimum is located when the incoming photon energy matches the polaron energy (dashed line), reflecting that the EIT is caused by the formation of a polaron-polariton with a small damping. Also, the width of the 
EIT minimum narrows with increasing interaction, which is caused by a decreasing polaron residue $Z_{P}$. The reason is that $Z_{P}$ by definition determines the overlap between the polaron state $\left|\psi_{P, \mathbf{k}-\mathbf{k}_{\mathrm{cl}}}\right\rangle$ and the plane wave $|c\rangle$ state. These effects reflect the difference between the polaron-polariton and the polariton, which would simply give rise to perfect EIT at the horizontal line $\delta=0$. For stronger interactions, however, Fig. 2(a) shows that the optical depth at the minimum is nonzero, and that the minimum position is shifted away from the polaron energy. This reflects that the polaron-polariton picture has broken down and that the interplay between light coupling and strong interactions produces a complex state with no well-defined quasiparticle. In the rest of the paper, we will derive and discuss in detail these as well as other intriguing results showing the imprints of many-body physics on light transmission.

\section{FIELD THEORY}

In order to develop a nonperturbative theory that can simultaneously account for strong-light matter coupling as well as atomic interactions, we introduce the imaginary time Green's function $\mathcal{G}(\mathbf{p}, \tau)=-\left\langle T_{\tau}\left\{\Psi_{\mathbf{p}}(\tau) \Psi_{\mathbf{p}}^{\dagger}(0)\right\}\right\rangle$, where $T_{\tau}$ denotes time ordering and $\Psi_{\mathbf{p}}=\left[\gamma_{\mathbf{p}}, e_{\mathbf{p}}, c_{\mathbf{p}-\mathbf{k}_{\mathrm{cl}}}\right]^{T}$. Due to the coupling between light and atoms, the Green's function is a $3 \times 3$ matrix, and we write in frequency space $\mathcal{G}^{-1}(\mathbf{p}, z)=$ $\mathcal{G}^{(0)}(\mathbf{p}, z)^{-1}-\Sigma(\mathbf{p}, z)$ as

$$
\mathcal{G}^{-1}(\mathbf{p}, z)=\left[\begin{array}{ccc}
z-c p & -g \sqrt{n} & 0 \\
-g \sqrt{n} & z-\epsilon_{\mathbf{p}}^{(e)}-\Sigma_{e e} & -\Omega \\
0 & -\Omega & z-\epsilon_{\mathbf{p}-\mathbf{k}_{\mathrm{cl}}}^{(c)}-\Sigma_{c c}
\end{array}\right] .
$$

The off-diagonal self-energies $\Sigma_{c e}=\Sigma_{e c}=\Omega$ and $\Sigma_{\gamma e}=$ $\Sigma_{e \gamma}=g \sqrt{n}$ (both real) give the light-matter couplings responsible for the EIT phenomena [4], which can be read off from the second line of Eq. (1). The diagonal self-energy $\Sigma_{e e}$ gives the decay of the $|e\rangle$ atom due to the coupling $g$ to the $|\gamma\rangle \otimes|b\rangle$ continuum described within Weisskopf-Wigner theory [27-29]. Finally, $\Sigma_{c c}=n \mathcal{T}$ describes the scattering of a $|b\rangle$ atom out of the condensate by a $|c\rangle$ atom, which is the dominant process leading to the formation of the Bose polaron [24]. The scattering matrix $\mathcal{T}$ is evaluated in the so-called ladder approximation accounting for repeated boson-impurity scattering. This approximation includes the two-body $|b\rangle-|c\rangle$ scattering giving rise to strong Feshbach interactions exactly, and it has turned out to be surprisingly accurate for impurities in atomic gases even for strong interactions [20-22]. The scattering matrix is given by $\mathcal{T}$,

$$
\mathcal{T}(\mathbf{p}, z)=\frac{\mathcal{T}_{\nu}}{1-\mathcal{T}_{\nu} \Pi(\mathbf{p}, z)},
$$

where

$$
\Pi(\mathbf{p}, z)=-\sum_{\mathbf{k}, i \omega_{v}} \mathcal{G}_{11}\left(\mathbf{k}, i \omega_{\nu}\right) \mathcal{G}_{c c}\left(\mathbf{p}-\mathbf{k}, z-i \omega_{\nu}\right)
$$

is the regularized propagator for a pair of $|b\rangle$ and $|c\rangle$ atoms in the presence of a BEC $[30,31]$. Here the $|c\rangle$-atom propagator

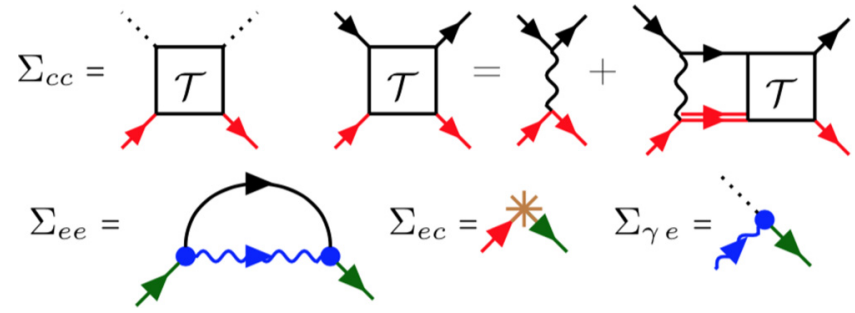

FIG. 3. Feynman diagrams representing our theory for light propagation in the presence of strong interactions. The self-energy for the $|c\rangle$ atoms describing strong $|b\rangle-|c\rangle$ interactions leading to polaron formation in the absence of light is shown in the top panel. The coupling to the classical and quantum light responsible for EIT in the absence of interactions is shown in the bottom panel.

$\mathcal{G}_{c c}$ is given by

$$
\begin{aligned}
& \mathcal{G}_{c c}^{-1}\left(\mathbf{p}-\mathbf{k}_{\mathrm{cl}}, \omega\right)=\mathcal{G}_{c c}^{(0)}\left(\mathbf{p}-\mathbf{k}_{\mathrm{cl}}, \omega\right)^{-1} \\
& \quad-\frac{|\Omega|^{2}}{\mathcal{G}_{e e}^{(0)}(\mathbf{p}, \omega)^{-1}-\Sigma_{e e}(\mathbf{p}, \omega)-n g^{2} \mathcal{G}_{\gamma \gamma}^{(0)}(\mathbf{p}, \omega)} .
\end{aligned}
$$

Since atom-light coupling is crucial for EIT physics, we have included the self-energies $\Sigma_{c e}$ and $\Sigma_{\gamma e}$ in the impurity propagator in Eq. (6), which describe the coupling to the excited state $|e\rangle$ and the photon $|\gamma\rangle$. This goes beyond the usual ladder approximation based on bare $c$ propagators or the equivalent variational Chevy ansatz, and it has important consequences as will be discussed in detail below. In Fig. 3 we illustrate the diagrams corresponding to Eq. (3). A dashed line is a $|b\rangle$ atom emitted from or absorbed into the BEC, a red line is the $|c\rangle$ propagator, a green line is the $|e\rangle$ propagator, a wavy blue line is the photon propagator, a black line is a $|b\rangle$ propagator, and a double solid red line corresponds to the impurity propagator including light-matter coupling. The classical field $\Omega$ is indicated by a $*$, a $\bullet$ is the dipole matrix element $g$ between the photons and the atoms, and a wavy black line is the $|b\rangle-|c\rangle$ interaction.

After diagonalizing Eq. (3), we obtain the photon Green's function describing light propagation in the BEC. Writing this as $\mathcal{G}_{\gamma \gamma}^{-1}(\mathbf{k}, \omega)=\epsilon \omega-c k$ allows us to relate the dielectric function $\epsilon=1+\chi$ and the optical susceptibility to the selfenergies as [32]

$$
\chi(\mathbf{k}, \omega)=-\frac{1}{c k} \frac{n g^{2}}{\omega-\epsilon_{\mathbf{k}}^{(e)}+i \Gamma_{e e}-\Omega^{2} \mathcal{G}_{P}\left(\mathbf{k}-\mathbf{k}_{\mathrm{cl}}, \omega\right)} .
$$

Here $\Gamma_{e e}=-\operatorname{Im} \Sigma_{e e}$ gives the decay rate of the excited state, and $\mathcal{G}_{P}^{-1}=\mathcal{G}_{c c}^{(0)^{-1}}-\Sigma_{c c}$; see Appendix A.

\section{POLARON-POLARITONS}

We can now show how the polaron-polariton emerges by ignoring the effects of light on the $|b\rangle-|c\rangle$ scattering described by the $\mathcal{T}$ matrix. As we shall see, this approximation is valid for $\Omega^{2} / \Gamma_{e e} \ll E_{n}$ where $E_{n}=k_{n}^{2} / 2 m$ sets the manybody energy scale. Then the term $\mathcal{G}_{P}$ in Eq. (7) becomes identical to the polaron Green's function in the ladder approximation [30], which has a pole at the undamped polaron ground state energy $E_{\mathbf{k}+\mathbf{q}-\mathbf{k}_{\mathrm{cl}}}^{(P)}+\delta$. Close to this pole, we can write $\mathcal{G}_{P}\left(\mathbf{k}+\mathbf{q}-\mathbf{k}_{\mathrm{cl}}, c k+\omega\right) \simeq Z_{P} /\left(\omega-E_{\mathbf{k}+\mathbf{q}-\mathbf{k}_{\mathrm{cl}}}^{(P)}-\delta\right)$, 
where $Z_{P}$ is the quasiparticle residue of the polaron wave function $\left|\psi_{P}\right\rangle$ introduced above. It follows from Eq. (7) that the on-shell susceptibility $\chi(\mathbf{k}, c k)$ vanishes at this pole, i.e., when $\mathcal{G}_{P}^{-1}\left(\mathbf{k}-\mathbf{k}_{\mathrm{cl}}, c k\right)=0$. Physically, this means that the photon can propagate undamped under perfect EIT conditions when its energy $c k$ matches that of a polaron with momentum $\mathbf{k}-\mathbf{k}_{\mathrm{cl}}$, i.e., when $c k=E_{\mathbf{k}-\mathbf{k}_{\mathrm{cl}}}^{(P)}+\epsilon_{c}+\omega_{\mathrm{cl}}$. In the wave function picture introduced above, this corresponds to light propagation carried by the polaron-polariton state $\left|D_{\mathbf{k}}^{P}\right\rangle$ given by Eq. (2), instead of the noninteracting polariton $\left|D_{\mathbf{k}}\right\rangle$.

To further explore many-body effects on the EIT spectrum, we use a pole expansion of $\mathcal{G}_{P}$ in Eq. (7). This gives

$$
\mathcal{G}_{\gamma \gamma}(\mathbf{k}+\mathbf{q}, c k+\omega) \simeq \frac{Z}{\omega-v_{g} q-\tilde{\delta}+i \frac{(\omega-\tilde{\delta})^{2}}{\sigma}},
$$

for the photon propagator around the EIT condition $\delta=$ $-E_{\mathbf{k}-\mathbf{k}_{\mathrm{cl}}}^{(P)}$ to first order in the deviations $\omega, \tilde{\delta}=\delta+E_{\mathbf{k}-\mathbf{k}_{\mathrm{cl}}}^{(P)}$, and $\mathbf{q}$, which is taken to be parallel to $\mathbf{k}$ for simplicity. We have neglected terms involving $\nabla_{\mathbf{k}} E_{\mathbf{k}-\mathbf{k}_{\mathrm{cl}}}^{(P)} \lesssim c_{s} \ll c$ and defined

$$
\begin{aligned}
& Z=\frac{1}{1+g^{2} n / \Omega_{P}^{2}}, \quad v_{g}=Z c, \\
& \sigma=\frac{\Omega_{P}^{2}}{\Gamma_{e e}}, \quad \Omega_{P}^{2}=Z_{P} \Omega^{2} .
\end{aligned}
$$

Here $Z=\cos ^{2} \theta$ is the residue of the EIT pole in the photon propagator, which in the wave function formulation is simply given by the photon component of the polaron-polariton state $\left|D_{\mathbf{k}}^{P}\right\rangle$ given by Eq. (2). Also, $v_{g}$ is the group velocity of light, $\sigma$ is the width of the EIT window, and $\Omega_{P}$ is the Rabi frequency renormalized by many-body correlations. From Eq. (9), we see in addition to moving the condition for EIT away from $\delta=0$, the formation of the polaron decreases both the group velocity of light in the BEC and the width of the EIT window through its residue $Z_{P}<1$.

We return to Fig. 2 showing the optical depth of a BEC of length $L$ as a function of the $|b\rangle-|c\rangle$ scattering length $a$ and the detuning $\delta$. The transmission is described by the optical depth

$$
\mathrm{OD}=\frac{\Gamma_{\gamma} k L}{v_{g}}=\operatorname{Im} \chi k L \quad \text { where } \Gamma_{\gamma}=Z c \operatorname{Im} \chi
$$

is the damping rate of the photons. The optical depth $\mathrm{OD}_{0}=$ $n g^{2} L / \Gamma_{e e} c$ in the absence of the classical control field serves as a reference. To demonstrate that the physics we discuss is within experimental reach, we consider the $4^{2} S_{1 / 2}$ to $4^{2} P_{1 / 2}$ transition in ${ }^{39} \mathrm{~K}$, which has already been employed in recent EIT and polaron experiments [20,33]. For this transition, $\Gamma_{e e}=\pi \times 2.978 \mathrm{MHz}$ corresponding to a wavelength $\lambda=2 \pi / k \simeq 700.1 \mathrm{~nm}$. Taking a typical BEC density of $n=2 \times 10^{14} \mathrm{~cm}^{-3}$, this gives $E_{n}=k_{n}^{2} / 2 m \simeq 420 \mathrm{kHz}$, and using $g^{2}=3 \pi c \Gamma_{e e} / k^{2}$ from Weisskopf-Wigner theory yields $\sqrt{n} g \simeq 6.1 \times 10^{5} E_{n}$. In order to resolve many-body physics in the spectrum, we choose a classical light coupling $\Omega$ so that the width $\sigma \simeq \Omega^{2} / \Gamma_{e e}=518 \mathrm{kHz}$ is comparable to $E_{n}$. Finally, the impurity momentum $\mathbf{k}-\mathbf{k}_{\mathrm{cl}}$, the temperature and the one-photon detuning $\Delta=\varepsilon_{\mathbf{k}}^{(e)}-c k$ are all zero. We also plot as dashed lines in Fig. 2(a) the attractive and repulsive polaron energies in the absence of light, determined by the pole of $\mathcal{G}_{P}$ with no light, i.e., $\Omega=0$. Figure 2(a) clearly demonstrates that the optical depth essentially vanishes when the two-photon detuning matches the polaron energy, $-\delta=$ $E_{\mathbf{k}-\mathbf{k}_{\mathrm{cl}}}^{(P)}$, for weak attractive coupling $1 / k_{n} a \lesssim-1$. This corresponds to the formation of a polaron-polariton leading to EIT as described above.

Figure 2(b) shows vertical cuts for several values of the interaction strength, and the vertical lines correspond to the polaron energy in the absence of any light. We see that the optical depth at the EIT resonance is in general larger for $k_{n} a>0$ compared to the attractive side, reflecting that the repulsive polaron is not the ground state so that it can decay into lower-lying states such as the Feshbach molecule even in the absence of light. In addition, we see an interesting doubledip structure in the optical depth for strong interactions $0 \lesssim$ $1 / k_{n} a \lesssim 1$. This is a genuine many-body effect beyond the quasiparticle picture: It is caused by a continuum of states involving Bogoliubov excitations of the BEC, which increases the transparency of the BEC for detunings away from the polaron energies.

\section{LIGHT-INDUCED DAMPING}

From Figs. 2(a)-2(b), we also see that for stronger interactions, the optical depth increases at the minimum, which moreover is shifted away from the polaron energy. As the interaction $k_{n}|a|$ increases, the EIT minimum is displaced away from the polaron energy, and the optical depth increases becoming substantial at unitarity $1 / k_{n} a=0$. This is caused by the interplay between the scattering and the light coupling, which leads to additional decay and eventual breakdown of the polaron, even when it is the ground state in the absence of light. The key point is that while the coupling $\Omega$ of the $|c\rangle$ state to the lossy $|e\rangle$ state is suppressed for the EIT resonant momentum $\mathbf{k}-\mathbf{k}_{\mathrm{cl}}$, it can be significant for other momenta where the photon is off-resonant. The remaining light coupling to the $|e\rangle$ state is controlled by the ratio $\Omega / \Gamma_{e e}$ and leads to damping of the impurity. This is of course irrelevant for EIT physics in the absence of interactions where the impurity momentum is fixed to $\mathbf{k}-\mathbf{k}_{\mathrm{cl}}$ by the incoming light. In the presence of interactions, however, atom scattering changes the momentum of the impurity to values, where the state $|c\rangle$ couples strongly to the lossy $|e\rangle$ state and this damping mechanism kicks in. One can show that for $\Omega / \Gamma_{e e} \ll 1$, the resulting damping of the polaron with resonant momentum $\mathbf{k}-\mathbf{k}_{\mathrm{cl}}=0$ is $\Gamma_{P} \propto\left(1-Z_{P}\right) \Omega^{2} / \Gamma_{e e}$; see details in Appendix B. This in turn results in a damping of the photons and a corresponding nonzero minimal optical depth given, respectively, by

$$
\Gamma_{\gamma} \simeq \Gamma_{P}+\frac{\Gamma_{P}^{2} \Gamma_{e e}}{\left|\Omega_{P}\right|^{2}}, \quad \mathrm{OD}=\Gamma_{\gamma} \frac{L}{v_{g}} \propto \mathrm{OD}_{0}\left(1-Z_{P}\right),
$$

for $\Omega / \Gamma_{e e} \ll 1, \Omega^{2} / \Gamma_{e e} \ll E_{n}$ and $\sqrt{n} g \gg \Omega_{P}$; see Appendix B. Equation (11) relates the optical depth of the medium to the incoherent excitations forming the polaron, which have a spectral weight of $1-Z_{P}$. In this sense, it provides a profound link between the propagation of light and the quasiparticle properties of the polaron. This, combined with the fact that the position and value at the minimum of the optical depth are determined by the energy and residue of the polaron, respectively, demonstrates how strong light-matter 
(a)

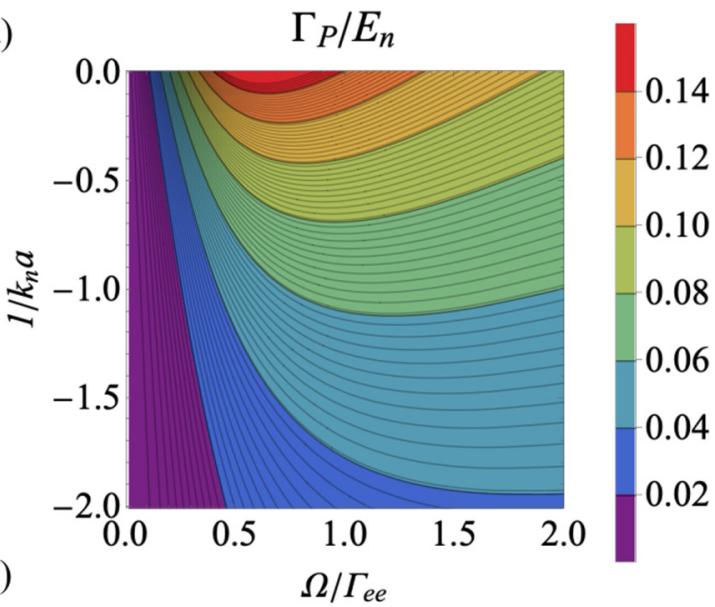

(b)

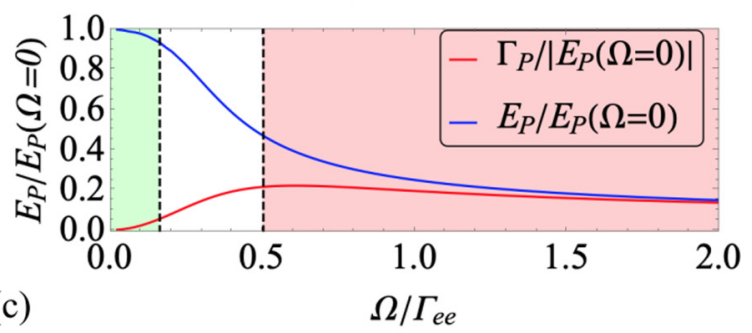

(c)

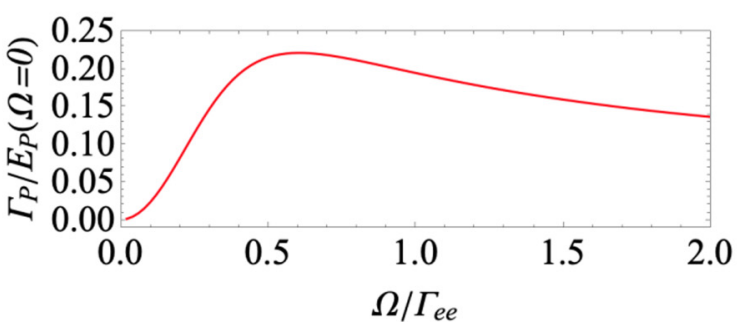

FIG. 4. (a) Decay rate of the impurity at the EIT minimum as a function of $\Omega / \Gamma_{e e}$ and $1 / k_{n} a$. (b) Polaron energy and damping as a function of $\Omega / \Gamma_{e e}$ at unitarity $1 / k_{n} a=0$. (c) The nonmonotonicity of the damping is illustrated.

coupling and slow light provides a powerful new platform for probing quantum many-body physics in a nondemolition scheme.

Increasing $\Omega$ further eventually makes the impurity states with momenta different from $\mathbf{k}-\mathbf{k}_{\mathrm{cl}}$ so strongly damped that scattering into them is suppressed. As a result, both the energy shift and the damping of the impurity with resonant momentum decreases, and the EIT spectrum approaches that of a ideal gas. In other words, interaction effects are suppressed for a strong control field giving rise to a nonmonotonic dependence of the damping and the eventual reemergence of the noninteracting polariton for large $\Omega$. This surprising effect can only be described using a nonperturbative theory taking into account the repeated scattering of impurities on the BEC; see Appendix A.

\section{REGIMES OF LIGHT PROPAGATION}

The different regimes of light propagation around the EIT minimum in the presence of interactions are shown in Fig. 4(a). It plots the damping rate of the impurity for the detuning $\delta$ giving the minimal optical depth as a function of the interaction strength $1 / k_{n} a$ and the classical light coupling $\Omega / \Gamma_{e e}$, keeping $\Gamma_{e e}$ fixed. All other parameters are as in Fig. 2. In agreement with the discussion above, we see that the damping of the impurity depends nonmonotonically on $\Omega / \Gamma_{e e}$ for fixed coupling strength $1 / k_{n} a$, as shown also in Fig. 4(c). For $\Omega / \Gamma_{e e} \ll 1$, the damping is small and light propagates in the form of a well-defined polaron-polariton giving rise to EIT with a small but finite residual absorption. The damping increases with increasing $\Omega / \Gamma_{e e}$ and it becomes substantial for strong coupling $1 / k_{n}|a| \gg 1$ and intermediate classical atom-light coupling $0.3 \lesssim \Omega / \Gamma_{e e} \lesssim 1$. Finally, both the decay and the energy shift of the impurity start to decrease for even stronger light coupling, and the ideal gas EIT spectrum governed by the noninteracting polariton reemerges. Note that this reemergence of ideal gas slow light propagation occurs for an arbitrarily large impurity-boson scattering length $a$, since scattering is suppressed into lossy $|c\rangle$ states with off-resonant momentum $\neq \mathbf{k}-\mathbf{k}_{\mathrm{cl}}$.

To illustrate this in more detail, we plot in Fig. 4(b) the polaron energy $E_{P}$ and its decay rate $\Gamma_{P}$ at unitarity $1 / k_{n} a=$ 0 as a function of $\Omega / \Gamma_{e e}$, in units of the polaron energy $E_{P}(\Omega=0)$ in the absence of light. The damping initially increases as $\Omega^{2}$ in agreement with the analysis above, and it is much smaller than the energy shift for $\Omega / \Gamma_{e e} \ll 1$ so that the polaron-polariton is well defined. For intermediate values $0.3 \lesssim \Omega / \Gamma_{e e} \lesssim 1$, however, the damping is of the same order as the energy, which is significantly shifted away from the polaron energy in the absence of light. In this region, the quantum state is a complex mixture of light and photons with no well-defined quasiparticle resulting in significant decay. Finally, both the energy shift and the damping become small for larger values of $\Omega / \Gamma_{e e}$, so that the noninteracting darkstate polariton emerges.

\section{CONCLUDING REMARKS}

We developed a nonperturbative theory for light propagation through a Bose-Einstein condensate in the presence of strong interactions, which permits us to explore the interplay of particle correlations and strong light-matter coupling. We have shown that the associated competition between the formation of polaritonic and polaronic quasiparticles can be observed and probed directly via the transmission spectrum of the interacting medium. This includes large deviations from noninteracting EIT as well as light-induced damping, and it offers a powerful nondestructive setup to manipulate and probe many-body physics. The presented approach can straightforwardly be generalized to include other interaction effects between the atomic states, or to describe other systems such as exciton-polaritons in semiconductors [17,18,34]. It therefore provides a powerful framework for describing systems with light-matter coupling in the presence of strong interactions and enables future explorations into key problems such as generating strong photon nonlinearities by polaronpolaron interactions [35].

\section{ACKNOWLEDGMENTS}

We thank Jan Arlt and Luis Ardila for helpful discussions. A.C.-G and G.M.B. acknowledge financial support 
from the Villum Foundation and the Independent Research Fund Denmark-Natural Sciences via Grant No. DFF-802100233B. This work was supported by the EU through the H2020-FETOPEN Grant No. 800942640378 (ErBeStA), by the DFG through Grant No. SPP1929, and by the DNRF through a Niels Bohr Professorship to T.P.

\section{APPENDIX A: SCATTERING MATRIX AND GREEN'S FUNCTIONS}

The Dyson's equation yields for the excited state

$$
\begin{aligned}
\mathcal{G}_{e e}^{-1}(\mathbf{p}, \omega)= & \mathcal{G}_{e e}^{(0)}(\mathbf{p}, \omega)^{-1}-n g^{2} \mathcal{G}_{\gamma \gamma}^{(0)}(\mathbf{p}, \omega) \\
& -|\Omega|^{2} \mathcal{G}_{P}\left(\mathbf{p}-\mathbf{k}_{\mathrm{cl}}, \omega\right)-\Sigma_{e e}(\mathbf{p}, \omega),
\end{aligned}
$$

where $\Sigma_{e e}(\mathbf{p}, \omega)$ accounts for the Lamb shift and the decay of the excited state, while $G_{P}$ is given in the main text.

Finally, the Dyson's equation for the photon field gives $\mathcal{G}_{\gamma \gamma}^{-1}(\mathbf{k}, \omega)=\epsilon(\mathbf{k}, \omega) \omega-c k$, and relates the Green's functions and self-energies in Fig. 3 to the optical susceptibility $\chi(\mathbf{k}, \omega)$ via $\epsilon(\mathbf{k}, \omega)=1+\chi(\mathbf{k}, \omega)$ [32] The expression for $\chi(\mathbf{k}, \omega)$ is provided in the main text.

Here we provide more details concerning the light-induced damping of the polaron discussed in the main text. This damping enters via the impurity states with momenta differing from $\mathbf{k}-\mathbf{k}_{\mathrm{cl}}$ inside the scattering matrix in Eq. (4). Let the momentum of the impurity inside the scattering matrix be $\mathbf{p}-\mathbf{k}_{\mathrm{cl}}$ and define $\mathbf{q}=\mathbf{p}-\mathbf{k}$. When $c q \ll n g^{2} / \Gamma_{e e}$, it follows from Eq. (6) that

$$
\begin{aligned}
\mathcal{G}_{c c}^{-1}(\mathbf{q}, c k+\omega) & \approx \mathcal{G}_{c c}^{(0)}\left(\mathbf{p}-\mathbf{k}_{\mathrm{cl}}, \omega\right)^{-1}-c q \frac{\Omega^{2}}{n g^{2}} \\
& \approx \mathcal{G}_{c c}^{(0)}\left(\mathbf{p}-\mathbf{k}_{\mathrm{cl}}, \omega\right)^{-1}-v_{g} q
\end{aligned}
$$

where we have used $v_{g} \simeq c \Omega^{2} / g n^{2}$. Equation (A2) shows that the impurities with momenta close to the resonant momentum $\mathbf{k}-\mathbf{k}_{\mathrm{cl}}$ are only weakly coupled to the excited state and thus have a long lifetime. The linear dispersion leads to an additional source of decay, Cherenkov radiation.

For $c q \gg n g^{2} / \Gamma_{e e}$ on the other hand, we get from Eq. (6)

$$
\mathcal{G}_{c c}^{-1}(\mathbf{q}, c k+\omega) \approx \mathcal{G}_{c c}^{(0)}\left(\mathbf{p}-\mathbf{k}_{\mathrm{cl}}, \omega\right)^{-1}-\frac{\Omega^{2}}{\omega-\epsilon_{\mathbf{k}+\mathbf{q}}^{(e)}+i \Gamma_{e e}}
$$

That is, for momenta far away from the resonant momentum the impurity couples to the excited state, which results in decay.

To estimate how this decay of impurities with momenta different from $\mathbf{k}-\mathbf{k}_{\mathrm{cl}}$ gives rise to a decay of the polaron with resonant momentum $\mathbf{k}-\mathbf{k}_{\mathrm{cl}}$ via the interaction, we first consider the case $\Omega / \Gamma_{e e} \ll 1$ and $\Omega^{2} / \Gamma_{e e} \ll E_{n}$. Estimating the propagators inside the scattering matrix to be given by Eq. (A3) then yields

$\Gamma_{P} \simeq-Z_{P} \operatorname{Im} \Sigma_{P}\left(E_{\mathbf{k}-\mathbf{k}_{\mathrm{cl}}}^{(P)}+i \Omega^{2} / \Gamma_{e e}\right) \approx\left(1-Z_{P}\right) \Omega^{2} / \Gamma_{e e}$. (a)

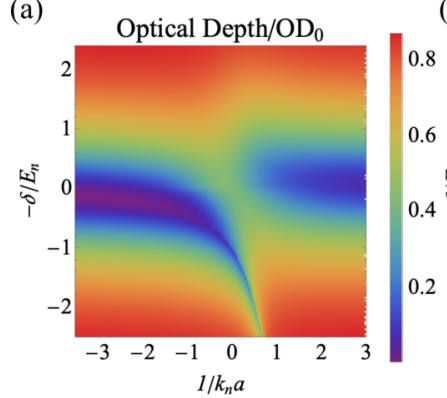

(b)

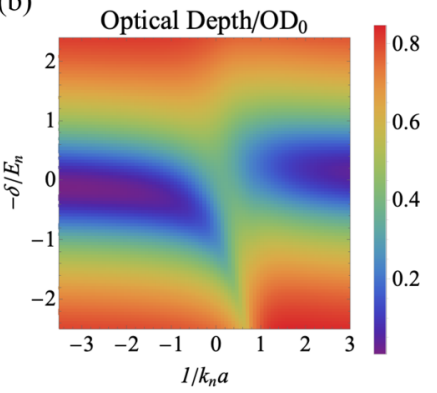

FIG. 5. (Left) Idealized picture of an undamped polaronpolariton. (Right) Physical polaron-polariton; here the light-matter coupling modifies the atomic scattering leading to deviations from the idealized undamped polaron-polariton picture.

In the opposite regime where $\Omega^{2} / \Gamma_{e e} \gg E_{P}$, the pair propagator in Eq. (4) can be approximated by $\Pi(\mathbf{p}, \omega) \propto$ $-i m^{3 / 2} \sqrt{\omega+i \Omega^{2} / \Gamma_{e e}}$. For $\Omega^{2} / \Gamma_{e e}$ larger that the typical atomic energies, this suppresses the boson-impurity scattering matrix in in Eq. (4) and thereby the impurity self-energy. Thus, one recovers the noninteracting dark state polariton for large $\Omega / \Gamma_{e e}$.

To illustrate the imprints of the light on the atomic scattering, we neglect those in Fig. 5 (left) and compare to the physical case discussed in the main text. For illustration purposes, we show the latter in Fig. 5 (right), which fully includes the light-matter coupling. In Fig. 5 (left) the optical depth at resonance $\delta=-E_{P}$ is strictly zero, illustrating that the ground-state polaron in the absence of any light-matter coupling is undamped. In agreement with the theory, the width of the EIT is reduced, as a consequence of the normalized Rabi frequency $\Omega_{P}^{2}=Z_{P}|\Omega|^{2}$, which is decreased with the residue of the polaron $Z_{P}$. The idealized undamped polaron-polariton corresponds to $c k_{n} \gg n g^{2} / \Gamma_{e e}$ and $\Omega^{2} / \Gamma_{e e} \ll E_{n}$ where the scattered $|c\rangle$ states are effectively decoupled from the resonant photons and the classical control field. In this limit, the atomic interactions can be described by the scattering matrix in the absence of any light-coupling [30].

\section{APPENDIX B: DAMPING OF THE POLARON-POLARITON}

The damping of the polaron in turn gives rise to a damping of the polaron-polariton given by

$$
\Gamma_{\gamma}=\frac{\tilde{Z} n g^{2} \Gamma_{P}}{\Gamma_{P} \Gamma_{e e}+\left|\Omega_{P}\right|^{2}}
$$

where

$$
\tilde{Z}=\frac{1}{1+\frac{n g^{2}\left(\left|\Omega_{P}\right|^{2}+\Gamma_{P}^{2}\right)}{\left(\left|\Omega_{P}\right|^{2}+\Gamma_{e e} \Gamma_{P}\right)^{2}}}
$$

is the modified residue of the EIT pole due to the light coupling. For $\left|\Omega_{P}\right|^{2} \ll \Gamma_{P}$ and taking $n g^{2} \gg\left|\Omega_{P}\right|^{2}$, the decay of the photon is $\Gamma_{\gamma}=\Gamma_{P}\left(1+\Gamma_{P} \Gamma_{e e} /\left|\Omega_{P}\right|^{2}\right)$. The optical depth $\mathrm{OD} \propto \mathrm{OD}_{0}\left(1-Z_{P}\right)$ can be obtained by using Eq. (A4) in Eq. (B1). 
[1] R. Lechner, C. Maier, C. Hempel, P. Jurcevic, B. P. Lanyon, T. Monz, M. Brownnutt, R. Blatt, and C. F. Roos, Phys. Rev. A 93, 053401 (2016).

[2] Y. Wang, S. Subhankar, P. Bienias, M. Łącki, T.-C. Tsui, M. A. Baranov, A. V. Gorshkov, P. Zoller, J. V. Porto, and S. L. Rolston, Phys. Rev. Lett. 120, 083601 (2018).

[3] Y.-F. Hsiao, P.-J. Tsai, H.-S. Chen, S.-X. Lin, C.-C. Hung, C.-H. Lee, Y.-H. Chen, Y.-F. Chen, I. A. Yu, and Y.-C. Chen, Phys. Rev. Lett. 120, 183602 (2018).

[4] M. Fleischhauer and M. D. Lukin, Phys. Rev. Lett. 84, 5094 (2000).

[5] D. F. Phillips, A. Fleischhauer, A. Mair, R. L. Walsworth, and M. D. Lukin, Phys. Rev. Lett. 86, 783 (2001).

[6] L. V. Hau, S. E. Harris, Z. Dutton, and C. H. Behroozi, Nature (London) 397, 594 (1999).

[7] U. Schnorrberger, J. D. Thompson, S. Trotzky, R. Pugatch, N. Davidson, S. Kuhr, and I. Bloch, Phys. Rev. Lett. 103, 033003 (2009).

[8] R. Zhang, S. R. Garner, and L. V. Hau, Phys. Rev. Lett. 103, 233602 (2009).

[9] J. D. Pritchard, D. Maxwell, A. Gauguet, K. J. Weatherill, M. P. A. Jones, and C. S. Adams, Phys. Rev. Lett. 105, 193603 (2010).

[10] J. J. Longdell, E. Fraval, M. J. Sellars, and N. B. Manson, Phys. Rev. Lett. 95, 063601 (2005).

[11] C. Chin, R. Grimm, P. Julienne, and E. Tiesinga, Rev. Mod. Phys. 82, 1225 (2010).

[12] I. Carusotto and C. Ciuti, Rev. Mod. Phys. 85, 299 (2013).

[13] F. Grusdt and M. Fleischhauer, Phys. Rev. Lett. 116, 053602 (2016).

[14] J. Thompson, T. Nicholson, Q.-Y. Liang, S. Cantu, A. Venkatramani, S. Choi, I. Fedorov, D. Viscor, T. Pohl, M. Lukin, and V. Vuletia, Nature (London) 542, 206 (2017).

[15] H. Busche, P. Huillery, S. W. Ball, T. Ilieva, M. P. A. Jones, and C. S. Adams, Nat. Phys. 13, 655 (2017).

[16] N. Stiesdal, J. Kumlin, K. Kleinbeck, P. Lunt, C. Braun, A. Paris-Mandoki, C. Tresp, H. P. Büchler, and S. Hofferberth, Phys. Rev. Lett. 121, 103601 (2018).
[17] N. Takemura, S. Trebaol, M. Wouters, M. T. Portella-Oberli, and B. Deveaud, Nat. Phys. 10, 500 (2014).

[18] M. Sidler, P. Back, O. Cotlet, A. Srivastava, T. Fink, M. Kroner, E. Demler, and A. Imamoglu, Nat. Phys. 13, 255 (2016).

[19] A. Maser, B. Gmeiner, T. Utikal, S. Götzinger, and V. Sandoghdar, Nat. Photon. 10, 450 (2016).

[20] N. B. Jørgensen, L. Wacker, K. T. Skalmstang, M. M. Parish, J. Levinsen, R. S. Christensen, G. M. Bruun, and J. J. Arlt, Phys. Rev. Lett. 117, 055302 (2016).

[21] M.-G. Hu, M. J. Van de Graaff, D. Kedar, J. P. Corson, E. A. Cornell, and D. S. Jin, Phys. Rev. Lett. 117, 055301 (2016).

[22] P. Massignan, M. Zaccanti, and G. M. Bruun, Rep. Prog. Phys. 77, 034401 (2014).

[23] M. Fleischhauer, A. Imamoglu, and J. P. Marangos, Rev. Mod. Phys. 77, 633 (2005).

[24] W. Li and S. Das Sarma, Phys. Rev. A 90, 013618 (2014).

[25] J. Levinsen, M. M. Parish, and G. M. Bruun, Phys. Rev. Lett. 115, 125302 (2015).

[26] Y. E. Shchadilova, R. Schmidt, F. Grusdt, and E. Demler, Phys. Rev. Lett. 117, 113002 (2016).

[27] V. Weisskopf and E. Wigner, Z. Phys. 63, 54 (1930).

[28] M. O. Scully and M. S. Zubairy, Quantum Optics (Cambridge University Press, Cambridge, 1997).

[29] It can also include additional decay processes for the $|e\rangle$ state in a phenomenological way if needed.

[30] S. P. Rath and R. Schmidt, Phys. Rev. A 88, 053632 (2013).

[31] R. S. Christensen, J. Levinsen, and G. M. Bruun, Phys. Rev. Lett. 115, 160401 (2015).

[32] G. Mahan, Many-Particle Physics (Kluwer Academic/Plenum Publishers, New York, NY, 2000).

[33] A. Lampis, R. Culver, B. Megyeri, and J. Goldwin, Opt. Express 24, 15494 (2016).

[34] J. Kasprzak, M. Richard, S. Kundermann, A. Baas, P. Jeambrun, J. M. J. Keeling, F. M. Marchetti, M. H. Szymańska, R. André, J. L. Staehli et al., Nature (London) 443, 409 (2006).

[35] A. Camacho-Guardian and G. M. Bruun, Phys. Rev. X 8, 031042 (2018). 